\title{
Optimized OFDM Model Using CMA Channel Equalization for BER Evaluation
}

\author{
Pratima Manhas ${ }^{\star 1}$, M.K Soni ${ }^{2}$ \\ ${ }^{1}$ FET, ECE Dept., Manav Rachna International University, Faridabad, India \\ ${ }^{2}$ ED\& Dean, FET, Manav Rachna International University, Faridabad, India \\ ${ }^{*}$ Corresponding author, e-mail: pratimamehak@gmail.com ${ }^{1}$, ed.fet@mriu.edu.in ${ }^{2}$
}

\begin{abstract}
Orthogonal Frequency Division Multiplexing (OFDM) is a type of Multicarrier Modulation (MCM) technique in which entire bandwidth is divided into large number of small sub-carriers and each subcarrier is transmitted parallel to achieve higher data rates. It has various applications like Digital Audio Broadcasting (DAB), Digital Video Broadcasting (DVB) and wireless LAN.OFDM technique is widely used in wireless communication system because of its very high data rate. The performance of FFT based OFDM system using Linear and cyclic channel coding and Constant Modulus Algorithm (CMA) equalizer is simulated using simulink model. The BER saving using the optimized proposed model with both linear and cyclic channel coding along with CMA equalizer is evaluated. The proposed work using cyclic channel coding with QPSK/QAM modulation and CMA as channel equalization under AWGN channel results in $52.6 \%$ and $96.3 \%$ BER reduction as compared to conventional OFDM model without channel coding, channel equalization and channel fading. So, CMA equalizer is used to enhance the performance of OFDM system.
\end{abstract}

Keywords: Multicarrier Modulation (MCM), Constant Modulus Algorithm (CMA), Digital Video Broadcasting (DVB), Digital Audio Broadcasting (DAB), subcarrier

\section{Introduction}

OFDM is an attractive modulation scheme with strongly efficient in bandwidth usage, immunity against multipath fading environment. It has less Inter Channel Interference (ICI), Inter Symbol Interference (ISI) and provides better spectral efficiency. OFDM has various merits over the conventional digital modulation techniques however, there are certain limitations of OFDM such as high Peak to Average Power Ratio (PAPR), high Bit Error Ratio (BER) and synchronization problem. In a wireless communication channel, the transmitted signal can travel from transmitter to receiver over multiple reflective paths. This gives rise to multipath fading which causes fluctuations in amplitude, phase and angle of arrival of the received signal. For example, the transmitted signal from the BTS (base transceiver station) may suffer multiple reflections from the buildings nearby, before reaching the mobile station. Such multipath fading channels are often classified into slow fading/fast fading and frequency-selective/flat fading channels. The performance of OFDM has been further enhanced by using various equalization techniques.

\section{Types of Equalizer}

Equalizer is used to reduce the intersymbol interference (ISI) at the receiver side. It can be classified as:

a. LMS Equalizer

Least mean squares (LMS) algorithms are a class of adaptive filter used to mimic a desired filter by finding the filter coefficients that relate to producing the least mean squares of the error signal (difference between the desired and the actual signal). The LMS Linear Equalizer block uses a linear equalizer and the LMS algorithm to equalize a linearly modulated baseband signal through a dispersive channel (Pandharipande, 2000).

b. RLS equalizer

The Recursive least squares (RLS) is an adaptive filter which recursively finds the coefficients that minimize a weighted linear least squares cost function relating to the input 
signals. This is in contrast to other algorithms such as the least mean squares (LMS) that aim to reduce the mean square error. In the RLS Linear Equalizer block uses a linear equalizer and the RLS algorithm to equalize a linearly modulated baseband signal through a dispersive channel.

c. CMA

CMA stands for constant modulus algorithm. The CMA Equalizer block uses a linear equalizer and the constant modulus algorithm (CMA) to equalize a linearly modulated baseband signal through a dispersive channel. During the simulation, the block uses the CMA to update the weights, once per symbol (Rinne et al; 1994). If the Number of samples per symbol parameter is 1 , then the block implements a symbol-spaced equalizer; otherwise, the block implements a fractionally spaced equalizer.

\subsection{Need of Equalizer}

Typically in wireless channel, let the received output symbol at time $k$ is represented as shown in equation (1)

$$
y(k)=h x(k)+v(k)
$$

where: $h$ represents flat fading channel coefficients

$x(k)$ represents transmitted symbol at time $k$

$v(k)$ represents noise sample at time $k$

So, $y(k)$ depends only on current transmitted symbol $x(k)$ as shown in equation (1) In some situation the received symbol is also given as:

$$
y(k)=h(0) x(k)+h(1) x(k-1)+v(k)
$$

where $x(k-1)$ represents transmitted symbol at time $(k-1)$ and $y(k)$ represents the current symbol.

Basically we observe that output symbol $y(k)$ at time $k$ depends not only on $x(k)$ but also on previous symbol $x(k-1)$. The $x(k-1)$ is interfering with current symbol $x(k)$ which results in intersymbol interference(ISI) which degrades the performance of the system. So, the process of removing ISI to improve the performance of system is called as channel equalization. The process of recovering $x(k)$ means transmitting signal is known as channel equalization.

For general scenario, the received symbol is represented as:

$$
y(k)=h(0) x(k)+h(1) x(k-1)+h(2) x(k-2)+\cdots \ldots \ldots \ldots \ldots+h(L-1) x(k-L+1)+v(k)
$$

In this eqn (3)," L" channel taps (coefficients) are used which are represented as $h(0), h(1) \ldots \ldots ., h(L-1)$

If " $L$ " increases then previous symbols interferes more and more effect of ISI increases. In order to recover $(k)$, equalization is used. For equalization of $x(k)$, with $\mathrm{L}=2$ (channel coefficients), let us employ

$y(k+2), y(k+1)$ and $y(k)$ where $\mathrm{r}=3$ symbols or 3 tap equalizer is used.

The system model for $L=2$ and $r=3$ are given as in equationns (4-6):

$$
\begin{aligned}
& y(k)=h(0) x(k)+h(1) x(k-1)+v(k) \\
& y(k+1)=h(0) x(k+1)+h(1) x(k)+v(k+1) \\
& y(k+2)=h(0) x(k+2)+h(1) x(k+1)+v(k+2)
\end{aligned}
$$

The vector model of the above mentioned equations are given as:

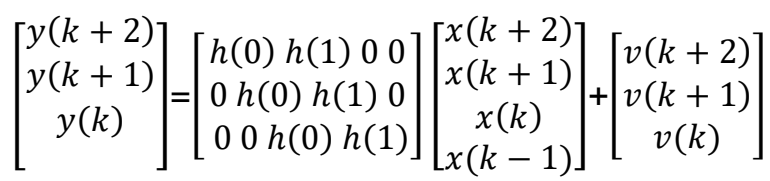


So, it is given as:

$$
\mathrm{y}(k)=\mathrm{H} x(k)+v(k)
$$

Let the equalizer weights be $c 0, c 1$ and $c 2$ where $\mathrm{C}$ is $3 \times 1$ equalizer vector.

$$
\mathrm{C}=\left[\begin{array}{l}
c 0 \\
c 1 \\
c 2
\end{array}\right]
$$

The equalizer equation is given as:

$=c 0 y(k+2)+c 1 y(k+1)+c 2 y(k)$ which is the weighted linear combination of $y(k+2), y(k+1)$ and $y(k)$.

The weights are designed $(c 0, c 1$ and $c 2)$ in such a way to minimize the interference (ISI) towards the detection of $x(k)$.

\section{Proposed Optimized Model Using CMA CHANNEL Equalization}

The model of OFDM using various equalizers is shown below in Figure 1. In this proposed work, the data is generated using Random integer and then integer to bit converter is used. After this the output of the converter is modulated by using digital modulation technique (BPSK, QPSK and QAM). Inverse fast Fourier transform is performed on the modulated signal and then the output of the IFFT is passed through multipath Rayleigh channel. The output of the channel is passed through various equalizer (LMS, RLS and CMA). Fast Fourier transform is performed on the equalized output and then it is demodulated. The demodulated output is passed through error rate calculator to determine the value of BER.The BER value is calculated for various equalization technique (LMS, RLS and CMA).A comparative analysis of $B E R$ is done for various equalization technique.

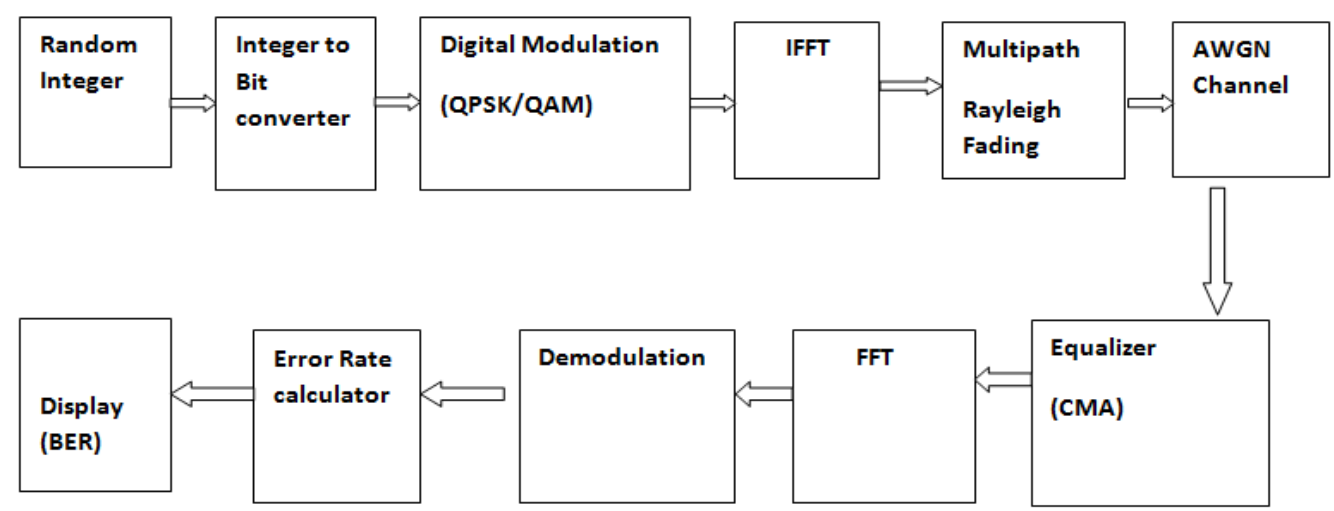

Figure 1. Proposed OFDM model using various equalizers (LMS/RLS/CMA) for different digital modulation

\subsection{Optimized OFDM Models}

So, the BER saving using the optimized proposed model with both linear and cyclic channel coding along with CMA equalizer has been given below in Table 1 and 2. The BER results of the optimized OFDM model using channel coding and CMA equalizer is shown in Figures 2-5. 


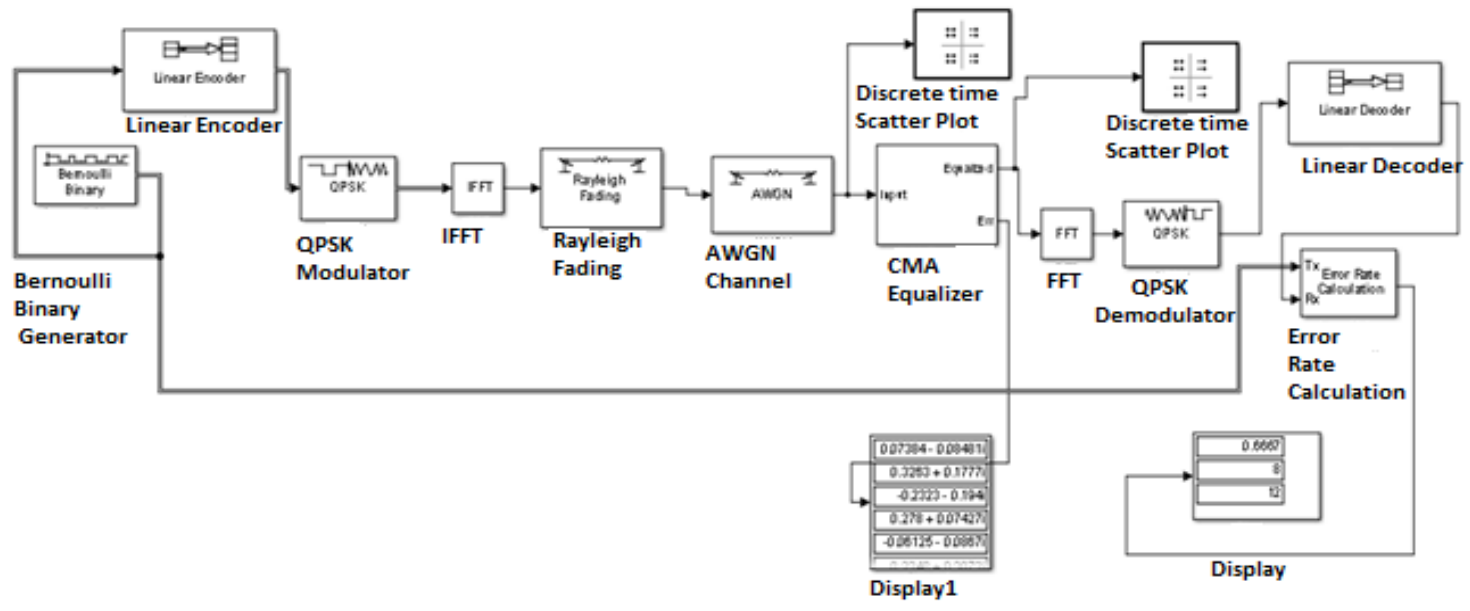

Figure 2. BER analysis of optimized OFDM model using CMA equalizer and QPSK modulation with Linear coding

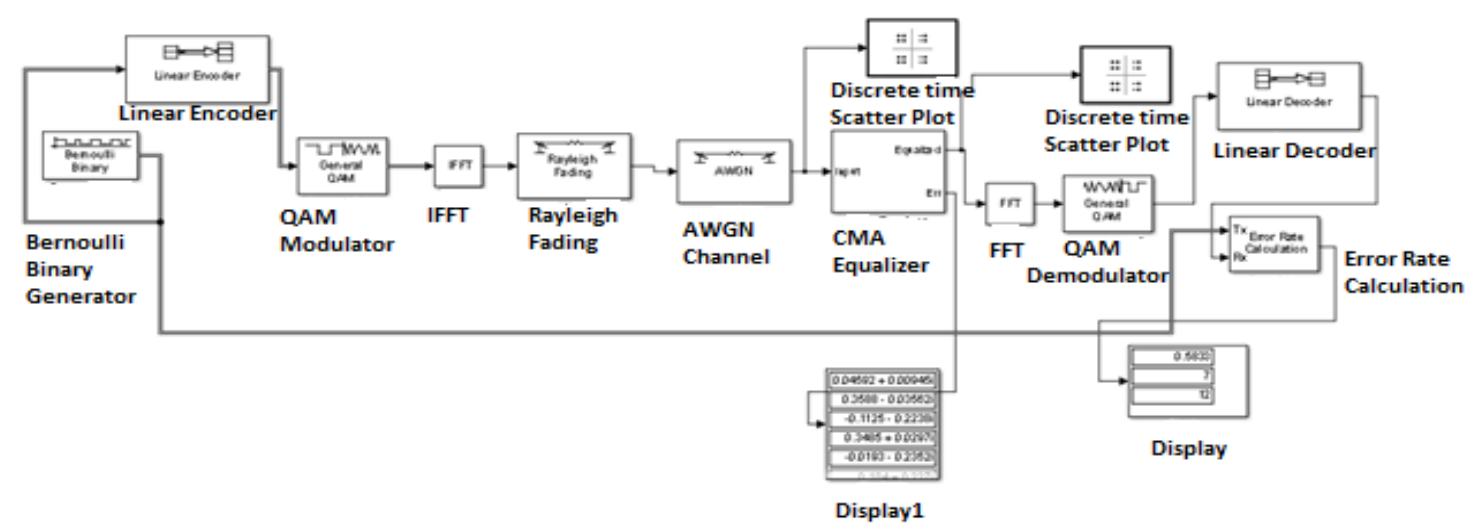

Figure 3. BER analysis of optimized OFDM model using CMA equalizer and QAM modulation with Linear coding

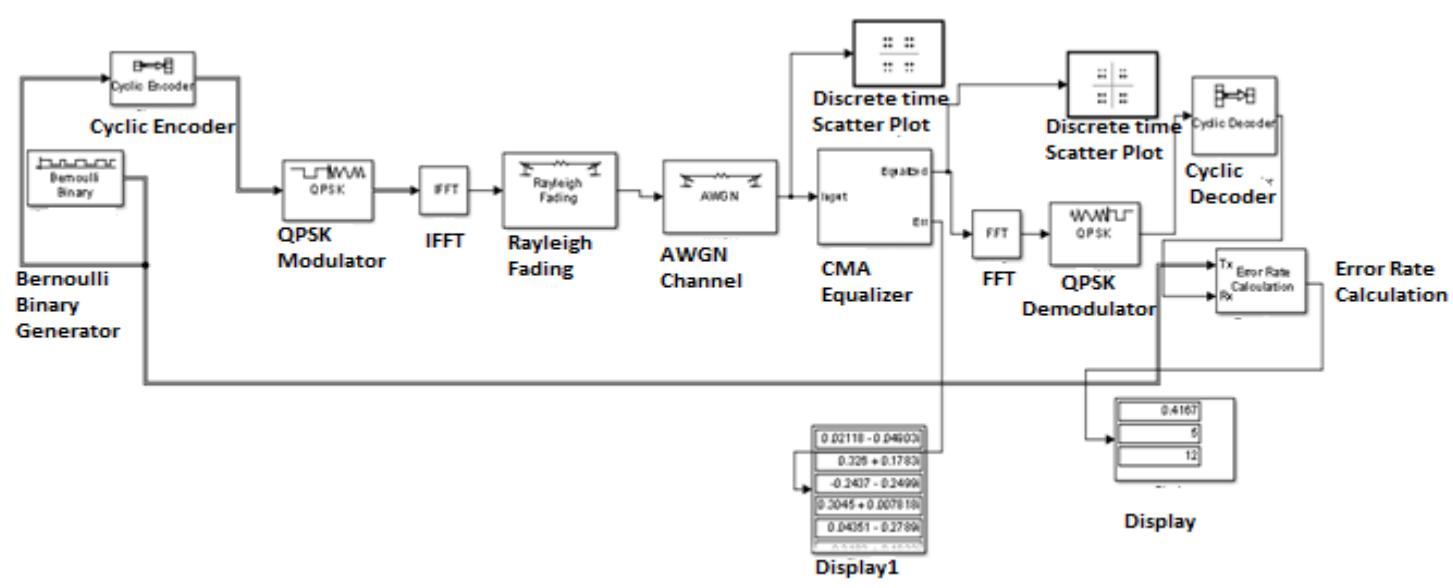

Figure 4. BER analysis of optimized OFDM model using CMA equalizer and QPSK modulation with cyclic coding 


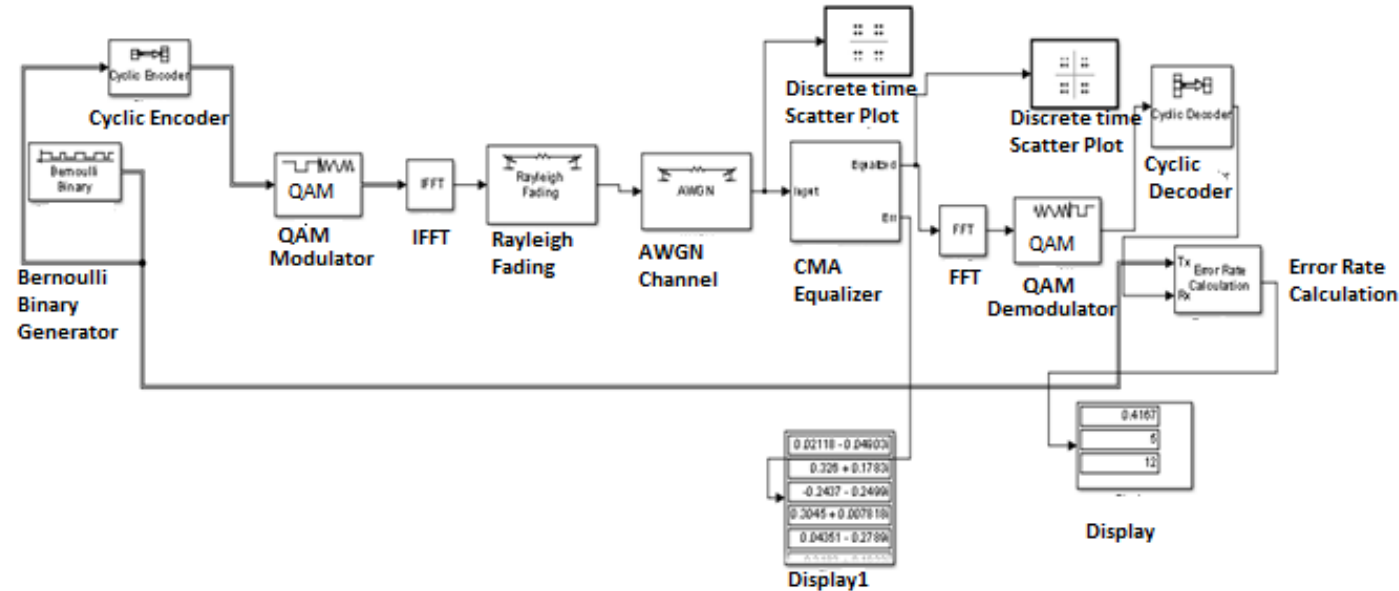

Figure 5. BER analysis of optimized OFDM model using CMA equalizer and QAM modulation with cyclic coding

The value of BER percentage saving using linear coding with CMA equalizer is shown in Table 1.

Table 1. BER Percentage Saving Using Linear Coding with CMA Equalizer

\begin{tabular}{cc}
\hline Digital Modulation & BER Percentage saving \\
\hline QPSK based OFDM system & 4.5 \\
QAM based OFDM system & 63.6 \\
\hline
\end{tabular}

The percentage saving value of BER with QPSK based OFDM system is 4.5 while in case of QAM its value is 63.6. The bar charts for the optimized model have been shown in Figures 6 and 7.

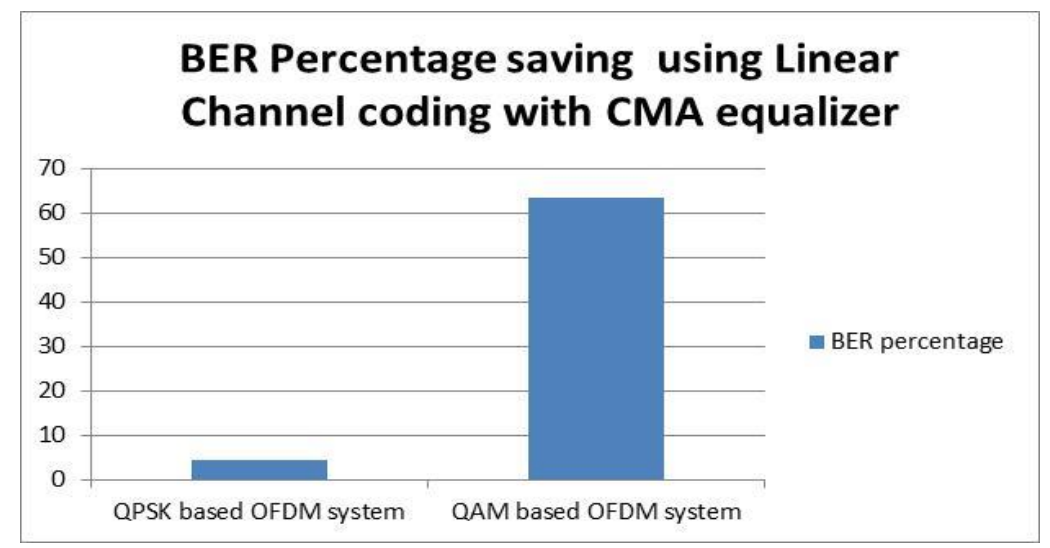

Figure 6. Bar chart for BER percentage saving using linear channel coding with CMA equalizer

The value of BER percentage saving using linear coding with CMA equalizer is shown in Table 2. The percentage saving value of BER with QPSK based OFDM system is 52.6 while in case of QAM its value is 96.3 . 
Table 2. BER Percentage Saving Using Cyclic Coding with CMA Equalizer

\begin{tabular}{cc}
\hline Digital Modulation & BER Percentage saving \\
\hline QPSK based OFDM system & 52.6 \\
QAM based OFDM system & 96.3
\end{tabular}

The bar chart of optimized OFDM model has been shown in Figure 7.

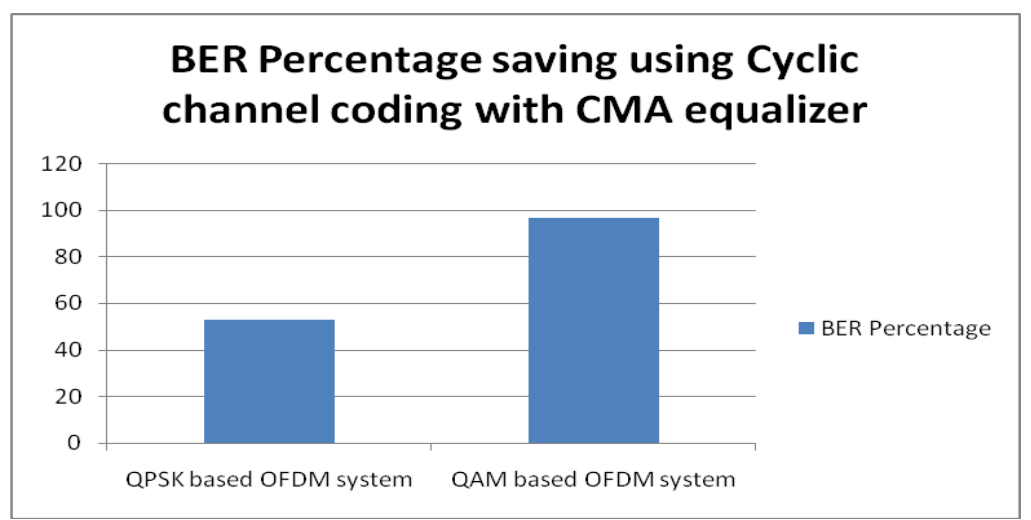

Figure 7. Bar chart for BER percentage saving using cyclic channel coding with CMA equalizer

The results shows that by using Cyclic coding with CMA equalizer, the BER value has been reduced.The proposed work using cyclic channel coding with QPSK/QAM modulation and CMA as a channel equalization under AWGN channel results in $52.6 \%$ and $96.3 \%$ BER reduction as compared to conventional OFDM model without channel coding, channel equalization and channel fading.

Hence, it has been concluded that by using various channel coding, different transform, channel Fading and channel equalization techniques results in reduction of BER and the performance of OFDM system has been improved.

\section{Conclusion}

The proposed work using cyclic channel coding with QPSK/QAM modulation and CMA as a channel equalization under AWGN channel results in $52.6 \%$ and $96.3 \%$ BER reduction as compared to conventional OFDM model without channel coding, channel equalization and channel fading. Hence, it has been concluded that by using various channel coding, different transform, channel Fading and channel equalization techniques results in reduction of BER and the performance of OFDM system has been improved.

\section{Acknowledgements}

The authors are very much thankful to the reviewers for their comments and suggestions to improve the quality of the manuscript.

\section{References}

[1] Nee RV, Prasad R. OFDM for Wireless Multimedia Communications. Artech House, Inc. 2000.

[2] Park M, Jun H, Cho J, Cho N, Hong D, Kang C. PAPR Reduction in OFDM transmission Using Hadamard Transform. In Communications, IEEE International Conference on 2000; 1: 430-433.

[3] Pandharipande A. Principles of OFDM. IEEE Potentials. 2000; 21(2): 16-19.

[4] Reddy, B, Lakshmi, B. 2013, September. Channel Estimation and Equalization in OFDM Receiver for WiMAX with Rayleigh Distribution. In Advanced Electronic Systems (ICAES), 2013 International Conference on IEEE. 2013: 337-339. 
[5] Rinne J, Renfors M. Equalization of Orthogonal Frequency Division Multiplexing Signals. In Global Telecommunications Conference, GLOBECOM'94, Communications: The Global Bridge, IEEE. 1994: 415-419.

[6] Robertson P, Kaiser S. Analysis of the Effects of Phase-noise in Orthogonal Frequency Division Multiplex (OFDM) Systems. In Communications, 1995. ICC'95 Seattle,'Gateway to Globalization, IEEE International Conference on IEEE. 1995; 3: 1652-1657.

[7] Schmidl TM, Cox DC. Robust Frequency and Timing Synchronization for OFDM. IEEE Transactions on Communications. 1997; 45(12): 1613-1621.

[8] Schulze H, Lüders C. Theory and Applications of OFDM and CDMA. Wideband Wireless Communications. John Wiley \& Sons. 2005.

[9] Sembiring Z, Syahruddin M. Performance Analysis of Discrete Hartley Transform Based OFDM Modulator and Demodulator. In Intelligent Systems, Modelling and Simulation (ISMS), Third International Conference on IEEE. 2012: 674-679.

[10] Sharma V, Shrivastav A, Jain A, Panday A. BER Performance of OFDM-BPSK,-QPSK,-QAM Over AWGN Channel Using Forward Error Correcting Code. International Journal of Engineering Research and Applications (IJERA). 2012; 2(3): 1619-1624. 